\title{
Floquet spin states in graphene under ac-driven spin-orbit interaction
}

\author{
A. López, ${ }^{1,2, *}$ Z. Z. Sun, ${ }^{1,3}$ and J. Schliemann ${ }^{1}$ \\ ${ }^{1}$ Institute for Theoretical Physics, University of Regensburg, D-93040 Regensburg, Germany \\ ${ }^{2}$ Centro de Física, Instituto Venezolano de Investigaciones Científicas, Apartado 21874, Caracas 1020-A, Venezuela \\ ${ }^{3}$ School of Physical Science and Technology, Soochow University, Suzhou, Jiangsu 215006, China
}

(Received 2 April 2012; published 15 May 2012)

\begin{abstract}
We study the role of periodically driven time-dependent Rashba spin-orbit coupling (RSOC) on a monolayer graphene sample. After recasting the originally $4 \times 4$ system of dynamical equations as two time-reversal related two-level problems, the quasienergy spectrum and the related dynamics are investigated via various techniques and approximations. In the static case, the system is gapped at the Dirac point. The rotating wave approximation (RWA) applied to the driven system unphysically preserves this feature, while the Magnus-Floquet approach as well as a numerically exact evaluation of the Floquet equation show that this gap is dynamically closed. In addition, a sizable oscillating pattern of the out-of-plane spin polarization is found in the driven case for states that are completely unpolarized in the static limit. Evaluation of the autocorrelation function shows that the original uniform interference pattern corresponding to time-independent RSOC gets distorted. The resulting structure can be qualitatively explained as a consequence of the transitions induced by the ac driving among the static eigenstates, i.e., these transitions modulate the relative phases that add up to give the quantum revivals of the autocorrelation function. Contrary to the static case, in the driven scenario, quantum revivals (suppressions) are correlated to spin-up (down) phases.
\end{abstract}

DOI: 10.1103/PhysRevB.85.205428

PACS number(s): 81.05.ue, 71.70.Ej, 72.25.Pn

\section{INTRODUCTION}

One of the key features of relativistic (massless) freeparticle states is that they evolve, at least in effectively one-dimensional situations, in time without spreading. This in turn relies on the linear nature of the relativistic dispersion relation, which for photons reads $\omega(k)=c k$, with $\mathrm{c}$ the speed of light. The condensed matter relativistic-particle analog is found in the low-energy approximation (long wavelength) of single-layer graphene where the chiral massless particles move with a speed $v_{F} \approx c / 300 .{ }^{1}$ This linear spectrum provides graphene with remarkable transport properties such as high mobility, ${ }^{2}$ Klein tunneling, ${ }^{3}$ and unconventional spin Hall effect. $^{4,5}$ The later stems from the interplay between intrinsic spin-orbit interaction and the coupling extrinsically induced by external gate voltages or an appropriate substrate. This extrinsic coupling, ${ }^{6-8}$ the so-called Rashba spin orbit (RSOC) has been also found to give rise to spin polarization ${ }^{9}$ and relaxation ${ }^{10,11}$ effects.

Although the role of static RSOC on graphene has been extensively discussed in the literature, to the best of our knowledge, the role of periodically driven time-dependent RSOC on graphene samples has not been analyzed so far. Yet, recent works have focused on the dynamical features of charge currents induced by means of time-dependent extrinsic spin-orbit interaction on mesoscopic semiconductor quantum rings where Rabi oscillations are shown to appear as well as collapse and revival phenomena. ${ }^{12}$ The main motivation of our work is twofold: first at all, we are interested in determining the feasibility of ac-driven fields to generate and modulate a finite spin polarization of carriers in graphene for states that under static conditions remain unpolarized. In addition, we are also interested in the dynamical modulation of the effective Rashba coupling strength $\Lambda=\lambda / \hbar \Omega$ that would allow to explore regimes beyond the static limit domain.
Taking advantage of the periodicity of the problem, the evolution equations can be solved via Floquet theory. A standard approach here consists of expressing the Hamiltonian in a Fourier mode expansion leading to an infinite-dimensional eigenvalue problem for the so-called quasienergies. ${ }^{13,14}$ This quasienergy spectrum carries nontrivial information on the topological nature of the system under study, ${ }^{15}$ and for semiconductor quantum wells with a zincblende structure, it has been recently shown that ac driving can induce a topological phase transition. ${ }^{16}$

Practically, in order to treat the infinite eigenvalue problem, one has to truncate at an order of the harmonic expansion chosen appropriately to yield well converged results. An alternative approach to the Floquet problem, which does not rely on Fourier expansions, has been devised by Magnus. ${ }^{17}$ This method appears to be somewhat less popular and amounts in formulating the time evolution operator as the exponential of a series of nested commutators. It has the virtue of both preserving unitarity at any order in the series expansion (in contrast to truncation of the Dyson series within a perturbative approach) and avoids the infinite-dimensional eigenvalue problems. Following Ref. 18, we will make use of the Magnus expansion approach combined with Floquet theory in order to generate semianalytical solutions of the dynamics induced by periodic RSOC.

Since RSOC couples the spin and pseudospin degrees of freedom the problem is, for a given wave vector, generically four dimensional. However, by an appropriate unitary transformation, the evolution equations can be recast as a set of two equivalent two-level Schrödinger equations related by time reversal. In this way, we can explicitly analyze which static states get dynamically coupled.

Our main results are the following. The ac driven RSOC induces a quasienergy spectrum where the original gap due to static spin-orbit coupling is dynamically closed. In particular, 
at the Dirac point $(\vec{k}=0)$, the dynamics is exactly solvable with zero quasienergy Floquet states. This quasienergy spectrum is twofold degenerate as a consequence of time reversal invariance of spin-orbit interaction, and the closing of the original gap is due to the destructive interference induced among the initially uncoupled positive and negative energy RSOC eigenstates. Then we show that sizable alternating out of plane spin polarization ensues on states that under static conditions remain unpolarized. We also find that the uniform interference pattern shown by the autocorrelation function for static RSOC gets distorted due to the interlevel mixing of the static eigenstates that dynamically modulates the relative phases that add up in the quantum revivals of the autocorrelation function. In the driven case, quantum revivals (suppressions) are directly correlated to spin-up (down) phases of the out-of-plane spin polarization. Since the autocorrelation function is related to the Fourier transform of the local density of states, ${ }^{19}$ and because spin probes can be more demanding in practical implementations than charge detection, its evaluation yields useful indirect information on the spin degree of polarization. We believe these findings have the potential to provide interesting new strategies to dynamically control spin properties of charge carriers in graphene for future spintronics applications.

The paper is organized as follows. In Sec. II, we describe the spectum for static RSOC and introduce the model Hamiltonian for periodically driven RSOC. Here, we also present the exact solution to the dynamical equations corresponding to the Dirac point $k=0$. The main results of the Floquet-Magnus approach for the semianalytical solution of the evolution operator at finite momentum are presented in Sec. III. Next, in Sec. IV, we compare the quasienergy spectrum obtained through Magnus approach to that given by making a rotating wave approximation. We also evaluate and discuss the outof-plane spin polarization as well as the onset of quantum revivals for the autocorrelation function. Finally, in Sec. V, we give some concluding remarks and discuss an experimental scenario where our results could be tested.

\section{MODEL}

We consider a graphene monolayer sample subject to periodic time-dependent spin-orbit interation of the Rashba type. In graphene, RSOC interaction emerges as a consequence of $\sigma$ and $\pi$ orbital mixing ${ }^{21}$ and stems from the induced electric field due to the substrate over which the graphene sample lies or by applied gate voltages. Then, a periodic modulation can, in principle, be implemented by means of time-dependent gate voltages or by the induced time varying electric field within a parallel plates capacitor coupled to a LC circuit. Under these circumstances, the induced RSOC perturbation could be given a periodic time dependence $V(t)=\lambda(t) \vec{s} \cdot(\hat{z} \times \vec{\sigma})$, where the driving amplitude will be assumed to be periodic $\lambda(t+T)=\lambda(t)$ with $\lambda(0)=\lambda_{R}$, the coupling strength in the static case.

Concerning energy scales, the value of the intrinsic and extrinsic spin-orbit coupling parameters $\Delta$ and $\lambda_{R}$ in graphene have been obtained by tight binding ${ }^{7,20}$ and band structure calculations. $^{6,21}$ They gave estimates in the range $10^{-6}-10^{-5} \mathrm{eV}$, much smaller than any other energy scale in the problem (kinetic, interaction, and disorder). However, the RSOC strength has recently been reported ${ }^{22}$ to be of order $\lambda_{R} \approx 0.2 \tau$, where $\tau \approx 2.8 \mathrm{eV}$ is the value of the first-neighbor hopping parameter for graphene within a tight-binding approach.

The formulation of the problem is as follows. In momentum space and taking into account the energy scales of spin-orbit coupling, we can work within the so-called long-wavelength approximation, where the total Hamiltonian for monolayer graphene in presence of time-dependent RSOC can be described by the $8 \times 8$ Hamiltonian $^{5}$

$$
\mathcal{H}(\vec{k}, t)=\left(\sigma_{x} \tau_{z} k_{x}+\sigma_{y} \tau_{y}\right) s_{0}+\lambda(t)\left(\sigma_{x} \tau_{z} s_{y}-\sigma_{y} s_{x}\right),
$$

where $v_{F} \sim 10^{6} \mathrm{~m} / \mathrm{s}$ is the Fermi velocity in graphene, $\vec{\sigma}=\left(\sigma_{x}, \sigma_{y}, \sigma_{z}\right)$ is a vector of Pauli matrices, with $\sigma_{z}=$ \pm 1 describing states on the sublattice $A(B)$ and so-called pseudospin degree of freedom, whereas $\tau_{z}= \pm 1$ describes the so-called Dirac points $\mathbf{K}$ and $\mathbf{K}^{\prime}$, respectively. In addition, $\vec{k}=\left(k_{x}, k_{y}\right)$ is the momentum measured from the $\mathbf{K}$ point and $s_{i}(\mathrm{i}=0, \mathrm{x}, \mathrm{y}, \mathrm{z})$ represents the real spin degree of freedom, with $s_{0}$ the identity matrix. In addition, $\lambda(t)$ gives the time dependence of the RSOC and we have neglected the intrinsic spin-orbit contributions. Now, since RSOC does not mix the valleys, we can focus on any of the two Dirac points, say $\mathbf{K}$, and then the results for the $\mathbf{K}^{\prime}$ point are found by the substitution $k_{x} \rightarrow-k_{x}$. Yet, we will formulate the problem in an isotropic way such that the results for the $\mathbf{K}^{\prime}$ Dirac point will immediately follow. Before dealing with the time-dependent problem, we summarize the main results for static RSOC.

The spectrum of the noninteracting Hamiltonian

$$
\mathcal{H}_{0}=\hbar v_{F} \vec{\sigma} \cdot \vec{k}
$$

is given by the linear dispersion relation

$$
\epsilon_{\sigma}^{0}(k)=\sigma \hbar v_{F} \sqrt{k_{x}^{2}+k_{y}^{2}} \equiv \sigma \hbar v_{F} k,
$$

whereas its eigenbasis is spanned by the spinors

$$
\left|\phi_{\sigma}(\vec{k})\right\rangle=\frac{1}{\sqrt{2}}\left(\begin{array}{c}
1 \\
\sigma e^{i \theta}
\end{array}\right)
$$

with $\tan \theta=k_{y} / k_{x}$ and $\sigma=1(-1)$ describes electron (hole) states. When a static RSOC interaction term is present, the Hamiltonian near the $\mathbf{K}$ point reads

$$
\mathcal{H}(\vec{k})=\hbar v_{F}\left(\sigma_{x} k_{x}+\sigma_{y} k_{y}\right) s_{0}+\lambda_{R}\left(\sigma_{x} s_{y}-\sigma_{y} s_{x}\right)
$$

and the energy spectrum changes to $\pm \epsilon_{ \pm}$with

$$
\epsilon_{ \pm}(k)= \pm \lambda_{R}+\sqrt{\lambda_{R}^{2}+\left(\hbar v_{F} k\right)^{2}} .
$$

Since RSOC mixes the $\sigma$ and $\pi$ atomic orbitals it induces a gap $\delta_{0}=2 \lambda$ at the Dirac point $k=0$. The static Rashba Hamiltonian in Eq. (5) is diagonalized by the unitary transformation $U(\vec{k})$ given explicitly as

$$
U(\vec{k})=\frac{1}{\sqrt{2}}\left(\begin{array}{ccccc}
-i e^{i \theta} \sin \gamma_{+} & -\cos \gamma_{+} & i \cos \gamma_{+} & e^{-i \theta} \sin \gamma_{+} \\
-i e^{i \theta} \sin \gamma_{-} & \cos \gamma_{-} & -i \cos \gamma_{-} & e^{-i \theta} \sin \gamma_{-} \\
i e^{i \theta} & \sin \gamma_{-} & -\cos \gamma_{-} & -i \cos \gamma_{-} & e^{-i \theta} \sin \gamma_{-} \\
i e^{i \theta} & \sin \gamma_{+} & \cos \gamma_{+} & i \cos \gamma_{+} & e^{-i \theta} \sin \gamma_{+}
\end{array}\right)
$$


where $\cos \gamma_{ \pm}=\epsilon_{ \pm} / \sqrt{\left(\hbar v_{F} k\right)^{2}+\epsilon_{ \pm}^{2}}$. In this basis, the static RSOC Hamiltonian reads

$$
\tilde{\mathcal{H}}(\vec{k})=\operatorname{Diag}\left\{-\epsilon_{+}, \epsilon_{-},-\epsilon_{-}, \epsilon_{+}\right\} .
$$

This particular choice of basis will simplify the calculations that follow.

We are interested in analyzing the emergent dynamics of Dirac fermions in monolayer graphene when the amplitude of RSOC is a periodically varying function of time $\lambda(t)=$ $\lambda_{R} \cos (\Omega t)$, with $\lambda_{R}$ and $\Omega$ the amplitude and frequency of the driving term. Then we would have to deal with the following $4 \times 4$ evolution equations:

$$
i \hbar \partial_{t} \Psi(\vec{k}, t)=\mathcal{H}(\vec{k}, t) \Psi(\vec{k}, t) .
$$

However, if we make use of the unitary transformation (7), the time-dependent Hamiltonian (1) becomes isotropic and block diagonal:

$$
\tilde{\mathcal{H}}(k, t)=\left(\begin{array}{cc}
h_{-}(k, t) & 0 \\
0 & h_{+}(k, t)
\end{array}\right)
$$

with both subblocks periodic functions of time, i.e., $h_{ \pm}(k, t+$ $T)=h_{ \pm}(k, t)$. Therefore the unitary transformation represented by Eq. (7) simplifies considerably the mathematical resolution of the evolution equations by recasting the problem as two time-reversal pairs of coupled $2 \times 2$ two-level problems. In addition, it has the physical appealing feature of clearly giving the subset of states that are dynamically coupled through the time-dependent interaction.

Let us then focus on the upper block $h_{-}(k, t)$ that reads

$$
\begin{aligned}
h_{-}(k, t)= & -\frac{2}{\epsilon_{-}+\epsilon_{+}} \\
& \times\left(\begin{array}{cc}
\left(\hbar v_{F} k\right)^{2}+\lambda(t) \epsilon_{+} & \hbar v_{F} k\left[\lambda_{R}-\lambda(t)\right] \\
\hbar v_{F} k\left[\lambda_{R}-\lambda(t)\right] & -\left(\hbar v_{F} k\right)^{2}+\lambda(t) \epsilon_{-}
\end{array}\right),
\end{aligned}
$$

whereas the lower block is obtained by changing the sign of the amplitude $\lambda_{R} \rightarrow-\lambda_{R}$. Because of this symmetry relation among the two subspaces, their quasienergy spectra are identical. This is to be expected since RSOC is time-reversal invariant.

First of all, we notice that in the static limit $\lambda(t) \rightarrow \lambda_{R}$ the reduced Hamiltonian (11) is block diagonal:

$$
h_{-}(k)=\left(\begin{array}{cc}
-\epsilon_{+} & 0 \\
0 & \epsilon_{-}
\end{array}\right) .
$$

We also note that at the Dirac point $k=0$, one gets

$$
h_{-}(0, t)=\left(\begin{array}{cc}
-2 \lambda(t) & 0 \\
0 & 0
\end{array}\right) .
$$

In this case, the resulting dynamics

$$
i \hbar \partial_{t}|\phi(t)\rangle=h_{-}(0, t)|\phi(t)\rangle
$$

is exactly solved by the eigenspinors

$$
\left|\phi_{1}(t)\right\rangle=\left(e^{2 i f(t)}, 0\right), \quad\left|\phi_{2}(t)\right\rangle=(0,1),
$$

where

$$
f(t)=\frac{1}{\hbar} \int_{0}^{t} d t^{\prime} \lambda\left(t^{\prime}\right)
$$

As will be discussed below, these solutions correspond to zero-quasienergy Floquet states. The corresponding evolution operator is diagonal and given as

$$
U_{-}(0, t)=e^{i f(t)} \operatorname{Diag}\left\{e^{i f(t)}, e^{-i f(t)}\right\} .
$$

\section{MAGNUS-FLOQUET APPROACH}

Although we have shown that at $k=0$, the dynamics is exactly solvable, this is no longer true for finite $k$. Then, we need to resort to approximate solutions. As we discuss below, a semianalytical approach known as Magnus-Floquet expansion will be suitable for dealing with the dynamical equations of periodically driven systems. Since the Magnus-Floquet approach is not so popular in the literature, we now briefly summarize its main results (see Ref. 18 for more detailed derivations).

In the language of differential equations, the matrix solution $S(t)$ of an $n$-dimensional system of dynamical evolution equations (here, we omit the orbital degrees of freedom for ease of notation),

$$
\partial_{t} \Psi(t)=A(t) \Psi(t)
$$

i.e., a matrix that satisfies

$$
\partial_{t} S(t)=A(t) S(t),
$$

is called a fundamental matrix solution if all its columns are linearly independent. If in addition, there is a time $t=t_{0}$ such that $S\left(t_{0}\right)$ is the identity matrix, then $S(t)$ is called a principal fundamental matrix solution. To solve Eq. (19), Magnus ${ }^{17}$ proposed to find exponential solutions to the evolution operator in the form

$$
S(t)=e^{M(t)}
$$

and then wrote $M(t)$ as an infinite series

$$
M(t)=\sum_{j=1}^{\infty} M_{j}(t),
$$

where each term $M_{j}(t)$ is given as a combination of nested commutators with the first terms reading as

$$
\begin{gathered}
M_{1}(t)=\int_{0}^{t} A\left(t_{1}\right) d t_{1}, \\
M_{2}(t)=\frac{1}{2} \int_{0}^{t} d t_{1} \int_{0}^{t_{1}}\left[A\left(t_{1}\right), A\left(t_{2}\right)\right] d t_{2}, \\
M_{3}(t)=\frac{1}{6} \int_{0}^{t} d t_{1} \int^{t_{1}} d t_{2} \int_{0}^{t_{2}}\left\{\left[A\left(t_{1}\right),\left[A\left(t_{2}\right), A\left(t_{3}\right)\right]\right]\right. \\
\left.+\left[A\left(t_{3}\right),\left[A\left(t_{2}\right), A\left(t_{1}\right)\right]\right]\right\} d t_{3}, \\
\vdots
\end{gathered}
$$

On the other hand, for periodic driving ${ }^{13,14} A(t+T)=$ $A(t)$, Floquet's theorem states that the principal fundamental solution of the dynamical equations can be written as

$$
S(t)=P(t) e^{t F},
$$


where $P$ and $F$ are $n \times n$ matrices, such that $P(t)$ is periodic $P(t+T)=P(t)$ and $F$ is time independent. Floquet's theorem is the time-dependent analog of Bloch's theorem in solid state physics for spatially periodic structures and it provides a time-dependent transformation such that the so-called Floquet states evolve according to the time-independent matrix $F$. This time-dependent transformation is implemented by $P(t)$.

One important remark is in order since although the interaction $A(t)$ is periodic, the corresponding evolution matrix $S(t)$ is not, i.e., $S(t+T) \neq S(t)$. In fact, $S(T)$ carries indeed nontrivial information on the dynamics of the periodic system. The eigenvalues of $F$ are called Floquet exponents $\rho$. These Floquet exponents can be found by diagonalizing $S(T)=e^{T F}$. Yet, they are not uniquely defined since $\rho \rightarrow \rho+2 i n \pi / T$ leaves $S(T)$ invariant.

In order to determine those exponents, one standard approach consists of performing an expansion in the (infinite) eigenbasis of time-periodic functions $\xi_{N}(t)=e^{i N \Omega t}$ (Fourier modes). In this periodic basis, the evolution operator is diagonalized and the Floquet exponents $q_{n}$ are the logarithms of the eigenvalues of the evolution operator evaluated at $t=T$, i.e., $S(T)$. Then, in order to deal with the infinite eigenvalue problem, one resorts to a truncation procedure in order to determine the Floquet exponents.

The Magnus approach avoids the need to solve the infinitedimensional eigenvalue problem and has the physical virtue of preserving unitarity of the evolved state to any order in the expansion. The connection between Magnus expansion and Floquet's theorem is found in Ref. 18 where the authors present a solution of the evolution equations that consists of writing the periodic part $P(t)$ as an exponential,

$$
P(t)=e^{\Omega(t)}, \quad \Omega(t+T)=\Omega(t),
$$

and then they proceed to expand both operators $\Omega(t)$ and $F$ in power series:

$$
\Omega(t)=\sum_{j=1}^{\infty} \Omega_{j}(t), \quad F=\sum_{j=1}^{\infty} F_{j} .
$$

Now, since $S(t)$ is by construction a principal fundamental matrix solution $P(T)=P(0)$ is the identity matrix and one gets for all values of $j$,

$$
F_{j}=M_{j}(T) / T \text {. }
$$

Introducing the Bernoulli numbers $B_{l}\left(B_{0}=1, B_{1}=-1 / 2\right.$, $\left.B_{2}=1 / 6, B_{4}=-1 / 30, \ldots\right)$ such that $B_{2 m+1}=0$ for $m \geqslant 1$, the exponent operator term contributions $\Omega_{j}(t)$ satisfy a recurrence relation in terms of two auxiliary time-dependent operators $W(t)$ and $T(t)$, according to the relations

$$
\partial_{t} \Omega_{j}(t)=\sum_{l=0}^{j-1} \frac{B_{l}}{l !}\left[W_{j}^{(l)}(t)+(-1)^{l+1} T_{j}^{(l)}(t)\right] \quad(n \geqslant 1) .
$$

In turn, the $W^{\prime} s$ and $T^{\prime} s$ are given through the iterative relations:

$$
W_{j}^{(l)}=\sum_{m=1}^{j-l}\left[\Omega_{m}, W_{j-m}^{(l-1)}\right] \quad(1 \leqslant l \leqslant j-1),
$$

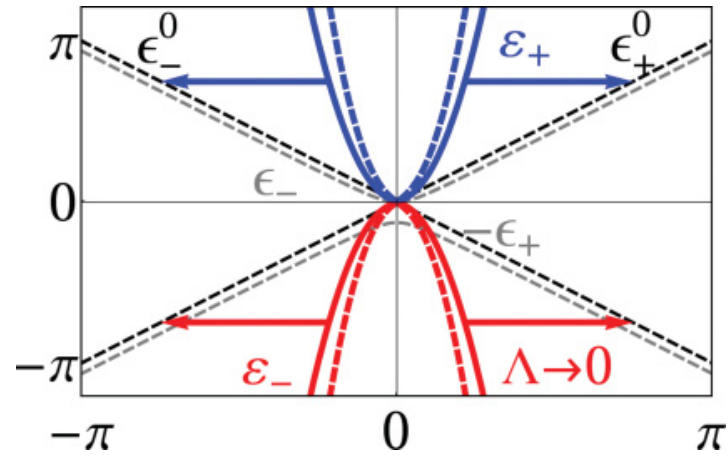

FIG. 1. (Color online) First Brillouin zone for the quasienergy spectrum as a function of adimensional noninteracting quasiparticle energy. Due to the dynamical interlevel mixing, the static gap $\delta_{0}$ gets closed (colored, thick continuous lines) as compared to the static interacting spectrum (gray, thin, dashed lines). Colored arrows depict the limit $\Lambda \rightarrow 0$ where the highly oscillatory contributions tend to cancel and the noninteracting spectrum is recovered (black, thin, dashed lines). The Floquet Fourier solutions (colored, thick, dashed lines) show qualitative agreement with the Magnus result, however, they converge slower for small $\Lambda$. We have expressed all quantities in units of $\tau=2.8 \mathrm{eV}$, the first-neighbor hopping parameter within a tight-binding approach and set $\Omega=1$.

$$
\begin{gathered}
T_{j}^{(l)}=\sum_{m=1}^{j-l}\left[\Omega_{m}, T_{j-m}^{(l-1)}\right] \quad(1 \leqslant l \leqslant j-1), \\
W_{1}^{(0)}=A, \quad W_{j}^{(0)}=0 \quad(j>1), \\
T_{j}^{(0)}=F_{j}, \quad(j>0) .
\end{gathered}
$$

In practical calculations, the relations in the last two lines serve to initialize the iterative procedure.

\section{RESULTS AND DISCUSSION}

In order to apply the previous results to our problem, one just has to make the following identifications $S(t) \rightarrow U_{-}(k, t)$, $A(t) \rightarrow-i h_{-}(k, t) / \hbar$. Then, the characteristic exponents are proportional to the quasienergies $\rho=-i q_{n}$.

For our calculation, we choose a time dependence of the form $\lambda(t)=\lambda_{R} \cos \Omega t$, where $\Omega$ is the frequency of the driving and $\lambda_{R}$ the RSOC strength and proceeded to evaluate $F_{j}$ by the iteration procedure described in the previous section.

We have found a characteristic behavior of the (adimensional) quasienergies $\varepsilon \pm=q_{ \pm} / \hbar \Omega$, which qualitatively do not change when one goes beyond third order in the Magnus-Floquet expansion (in Appendix we briefly describe the calculations up to fifth order). For finite $\Omega$, they explicitly read as

$$
\varepsilon_{ \pm}= \pm \sqrt{\kappa^{2}\left(16 \kappa^{2} \Lambda^{2}+\Lambda^{4}-2 \Lambda^{2}+1\right)},
$$

where we have defined the adimensional quantities $\kappa=$ $v_{F} k / \Omega$ and $\Lambda=\lambda_{R} / \hbar \Omega$.

In Fig. 1, we show the typical behavior of the quasienergies $\varepsilon_{ \pm}$(blue and red, thick continuous lines) given in Eq. (34) as a function of the adimensional quasiparticles noninteracting energy $\epsilon^{0}$. We also show (gray, thin, dashed lines) the corresponding static eigenvalues $\epsilon_{ \pm}$of the RSOC Hamiltonian 
as described by $h_{-}(k)$ as well as the eigenenergies of the noninteracting Hamiltonian (thin, dashed, black lines). We have also included (red and blue, thick, dashed lines) the result from a 20-mode Fourier expansion of the quasienergies. We have expressed all quantities in units of the first-neighbor hopping parameter $\tau=2.8 \mathrm{eV}$ and for finite $\Omega$, have set $\Omega=1$ and change the effective coupling $\Lambda$. This is true for all the figures within the dynamical case.

Since $h_{-}(k, t)$ mixes the static eigenstates of $h_{-}(k)$, the relative phases among them are dynamically modulated giving rise to interference phenomena, and we find that this leads to a dynamical closing of the original gap $\delta_{0}$. Therefore the exact solutions at $k=0$ correspond to vanishing quasienergies (modulo $\hbar \Omega$ ). We further find (see arrows in Fig. 1) that in the limit $\Lambda \rightarrow 0$, corresponding to a highly oscillating field, $\varepsilon_{ \pm} \rightarrow \epsilon_{ \pm}^{0}$ because then the influence of the driving quickly tends to vanish on average and intuitively one expects to recover the noninteracting linear spectrum. The result from a 20-mode Fourier expansion shows qualitative agreement to the Magnus-Floquet approach, however, a small discrepancy is found and the Magnus result converges faster in the limit of highly oscillating fields $\Lambda \rightarrow 0$, as depicted by thick arrows in Fig. 1.

Within the Magnus-Floquet approach, the evolution operator $U_{-}(\kappa, t)$ corresponding to the Hamiltonian $h_{-}(\kappa, t)$ is found to be given as

$$
U_{-}(\kappa, t)=e^{i f(t)} e^{i \vec{V}(\kappa, t) \cdot \vec{p}} e^{i \vec{v}(\kappa, t) \cdot \vec{p}},
$$

where $\vec{p}$ is a vector of Pauli matrices and the nonvanishing components of the vector $\vec{V}$ are given as

$$
\begin{gathered}
V_{x}(\kappa, t)=\frac{-\kappa \Lambda \sin \Omega t}{\sqrt{\kappa^{2}+\Lambda^{2}}}\left(4 \kappa^{2}-2 \Lambda^{2}+1+\Lambda^{2} \cos \Omega t\right), \\
V_{y}(\kappa, t)=2 \kappa \Lambda(1-\cos \Omega t), \\
V_{z}(\kappa, t)=\frac{\Lambda^{2} \sin \Omega t}{\sqrt{\kappa^{2}+\Lambda^{2}}}\left(6 \kappa^{2}+1-\kappa^{2} \cos \Omega t\right),
\end{gathered}
$$

whereas those of $\vec{v}$ are

$$
\begin{gathered}
v_{x}(\kappa, t)=\frac{t \kappa \Lambda}{\sqrt{\kappa^{2}+\Lambda^{2}}}\left(1+4 \kappa^{2}-\Lambda^{2}\right), \\
v_{z}(\kappa, t)=\frac{t \kappa^{2}}{\sqrt{\kappa^{2}+\Lambda^{2}}}\left(1-5 \Lambda^{2}\right) .
\end{gathered}
$$

At the Dirac point $\kappa=0$, we find that $v_{x}, v_{z}, V_{x}, V_{y}$ all vanish, whereas $V_{z}=\Lambda \sin \Omega t=f(t)$, and thus we recover the exact solution (17).

Before we proceed to evaluate other physical quantities of interest, we make a brief disgression on the importance of taking into account the full time dependence of the RSOC. In particular, due to smallness of the RSOC strength, one can try a rotating wave approximation (RWA) where for a given finite value of $k$ only near to resonance $\left(\Omega \sim 2 v_{F} k\right)$ terms are kept in the interacting Hamiltonian. Now we show that for the present model this approach gives unphysical results, for instance, it predicts a gap opening at $k=0$. This, in turn, would imply finite quasienergy mode contributions at the Dirac point, which contradicts the previously described exact result.
Since the results are easily found within the fourdimensional formulation, we briefly return to the original basis and make a full dimensional discussion. Within a RWA approach, the Hamiltonian reads (here, we omit the spatial degrees of freedom for ease of notation)

$$
\mathcal{H}^{\mathrm{RWA}}(t)=\mathcal{H}_{0}+i \lambda\left(\sigma_{+} s_{-} e^{i \Omega t}-\sigma_{-} s_{+} e^{-i \Omega t}\right),
$$

which for a given value of $k$ describes near resonance $\Omega \sim$ $2 v_{F} k$ spin and pseudospin flipping processes and neglects the so-called secular or counter-rotating terms that oscillate rapidly. In this case, the solution is exact and the adimensional quasienergy spectrum reads

$$
\varepsilon_{\sigma s}^{\mathrm{RWA}}=\frac{s}{2} \sqrt{\delta_{\mathrm{res}}^{2}+g_{\sigma}(\kappa)},
$$

where $\delta_{\text {res }}=2 \kappa-1$ describes the resonance and $g_{\sigma}(\kappa)=$ $\Lambda^{2}-\kappa+2 \sigma \sqrt{\kappa\left(\Lambda^{2}+1\right)+\Lambda^{4}}$. When we evaluate at $\kappa=0$ and finite $\Omega$ one would get the gaps $\Delta=\sqrt{\left(\Lambda^{2}+1 / 4\right.}$ for the originally gapped states and a dynamically opened gap on the static degenerate states $\Delta_{\text {dyn }}=1$. These results are in disagreement with the exact solution to the full equations (15) where we had found zero-energy solutions at $\kappa=0$, so this approach is not suitable to describe the dynamical features of the periodic driving. In Fig. 2, we depict the quasienergy spectrum as a function of noninteracting energies $\epsilon^{0}$ for this RWA solution.

After this brief discussion, we turn back our attention to the Magnus-Floquet solution in order to get additional information on the dynamical behavior induced on the system. This can be found by evaluating some other physical quantities of interest from an experimental point of view. First, we analyze the out-of-plane spin polarization $\mathcal{S}_{z}(\vec{k}, t)=\left\langle\Psi(\vec{k}, t)\left|S_{z}\right| \Psi(\vec{k}, t)\right\rangle$, where $S_{z}=\hbar / 2 \sigma_{0} \otimes s_{z}$ and $\sigma_{0}$ the identity matrix in pseudospin space. Using again the transformation (7), the outof-plane spin polarization reads $S_{z}(\vec{k})=U(k) S_{z} U^{\dagger}(\vec{k})$ and is found to be isotropic and block antidiagonal:

$$
S_{z}(\vec{k})=\left(\begin{array}{cc}
0 & s_{-} \\
s_{+} & 0
\end{array}\right)
$$

with $s_{ \pm}$given explicitly as

$$
s_{ \pm}=-\frac{\hbar}{2 \sqrt{\kappa^{2}+\Lambda^{2}}}\left(\kappa p_{0} \pm i \Lambda p_{y}\right)
$$

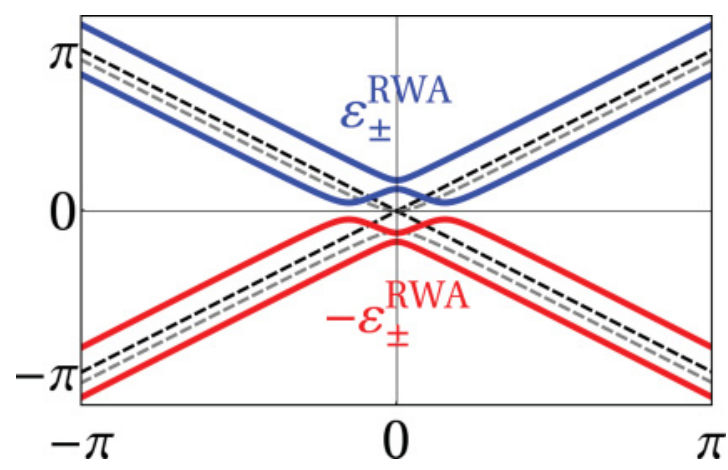

FIG. 2. (Color online) Quasienergies as a function of momenta. Gray continuous lines correspond to the static limit. The solution within this approach describes an unphysical gap opened at $\kappa=0$ (see main text). 


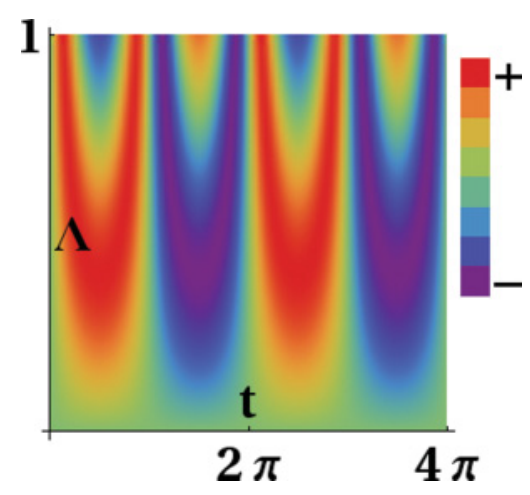

FIG. 3. (Color online) Density plot showing the out-of-plane spin polarization plotted against normalized time $t=\hbar / \tau$ for the exact solution at $\kappa=0$. The periodicity is inherited from the driving field. The vertical axis indicates the normalized adimensional field strength $\Lambda$ and one gets up (+) down (-) spin components phases equally separated by the zeros of $f(t)$.

with $p_{i}$ a vector of Pauli matrices and $p_{0}$ the two-dimensional identity matrix. The antidiagonal structure of $\tilde{S}_{z}$ in this basis reflects the fact that spin polarization is not conserved in presence of RSOC. Then, we have to evaluate

$$
\mathcal{S}_{z}(\vec{k}, t)=\left\langle\Psi(k, 0)\left|U^{\dagger}(k, t) S_{z}(k) U(k, t)\right| \Psi(k, 0)\right\rangle,
$$

for any initially prepared state $|\Psi(k, 0)\rangle$. Next, we separate explicitly the four spinor $|\Psi(k, 0)\rangle$ in upper and lower components as

$$
|\Psi(k, 0)\rangle=\frac{1}{\sqrt{2}}\left(\begin{array}{l}
\psi_{-} \\
\psi_{+}
\end{array}\right),
$$

where $\psi_{ \pm}$are normalized two-dimensional spinors. After some algebra, one gets for the spin polarization in terms of adimensional parameters:

$$
\mathcal{S}_{z}(\kappa, t)=\Re \psi_{-}^{*} e^{-2 i f(t)} e^{-i \vec{v} \cdot \vec{p}} e^{-i \vec{V} \cdot \vec{p}} s_{-} e^{i \vec{V} \cdot \vec{p}} e^{i \vec{v} \cdot \vec{p}} \psi_{+} / 2 .
$$

For a finite value of $\kappa$, we now choose the initial spinor configuration $\psi_{ \pm}=( \pm i, 1) / \sqrt{2}$, in such way that the out-ofplane polarization vanishes $\left\langle|\Psi(k, t)| S_{z}(k) \mid \Psi(k, t)\right\rangle=0$ for a static RSOC.

In Fig. 3, we show a density plot of the resulting outof-plane spin polarization for the exact solution $\kappa=0$. In this case, the only relevant parameter is the adimensional amplitude of the driving field $\Lambda$. As expected, for $\Lambda=0$, the system remains unpolarized for all values of the adimensional time $t$ (given in units of $\tau / \hbar$ ). For finite values of the effective coupling, an alternating pattern of spin phases (denoted as + and - representing up and down, respectively) are seen to appear as time evolves. They are symmetrically distributed among the values $t=n \pi$ where $f(t)$ and thus the relative phases among the static RSOC eigenstates vanish.

However, as shown in Fig. 4, once $\kappa$ is finite this panorama qualitatively changes. In this case, the additional interference due to level mixing induces a pattern of alternating maxima $(+)$ and minima $(-)$ for $t_{n}=n \pi, n=\in \mathbb{N}$. The reason for such a behavior is that for a given $t_{n}$ the evolution operator is given by $e^{T F}$, and thus the polarization maxima and minima $\mathcal{S}(\kappa, T)= \pm 1 / 2$ depend essentially on the quasienergy spectrum properties. Then, increasing $\Lambda$ makes these alternating

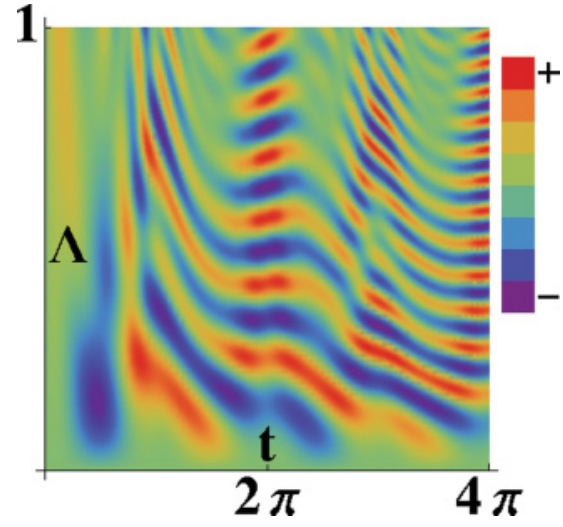

FIG. 4. (Color online) Density plot showing the time behavior of the out-of-plane spin polarization for the semianalytical MagnusFloquet solution at finite $\kappa=1$. Alternating maxima and minima appear due to interlevel mixing among different static eigenstates. This is also manifested by the loss of symmetry with respect to $t=\pi$ and stems from the driven modulated phases since dynamical interlevel mixing leads to interference effects.

maxima and minima to get closer. When we move to the next period (corresponding to $t=4 \pi$ in Fig. 4 ) the number of alternating maxima and minima is doubled, and so on. Therefore dynamical coupling produces a nonvanishing out-of-plane spin polarization with an oscillating time pattern resulting from the mixing of the static eigenstates such that the changing relative phases, modulated by the time-dependent interaction prevent total destructive interference to happen. Therefore ac-driven RSOC provides a suitable means to dynamically control the degree of spin polarization and could, in principle, serve to generate nonvanishing and nontrivial spin-polarized phases in otherwise unpolarized states in monolayer graphene.

In order to complement the just described physical picture of the spin polarization scenario, we evaluated the autocorrelation function $A(\vec{\kappa}, t)$. This is given by the projection of the evolved state $|\Psi(\vec{\kappa}, t)\rangle$ along a given (in principle, arbitrary) initial spinor configuration $|\Psi(\vec{\kappa}, 0)\rangle$, i.e.,

$$
A(\vec{\kappa}, t)=\langle\Psi(\vec{\kappa}, 0) \mid \Psi(\vec{\kappa}, t)\rangle .
$$

The absolute value of the autocorrelation function provides information on the so-called recurrences or quantum revivals of the dynamics, i.e., those values of the time parameter for which such overlapping is a maximum. In addition, its Fourier transform is proportional to the local density of states. ${ }^{19}$

In Fig. 5 (Fig. 6), we plot the absolute value of $A(\kappa, t)$ obtained by means of the exact (semianalytical) evolution operators. For the exact solution shown in Fig. 5, only large values of $\Lambda$ induce an appreciable phase change and the system remains mostly correlated to the initial state. As for the case of the out-of-plane spin polarization, for $t=t_{n}$, the autocorrelation gives maxima corresponding to red (dark gray) zones in the figure. These signal the return of the system to initial vanishing spin polarization. Maxima and minima of spin polarization correspond to partial quantum revivals. Given the definition of the autocorrelation as the probability that the system returns to its initial state, we find that for $\Lambda=0$ giving $\varepsilon=\kappa$, the loci of $A\left(\kappa, t_{n}\right)=1$ correspond to vanishing of spin polarization and are thus equally spaced as $\delta t=\pi$. 


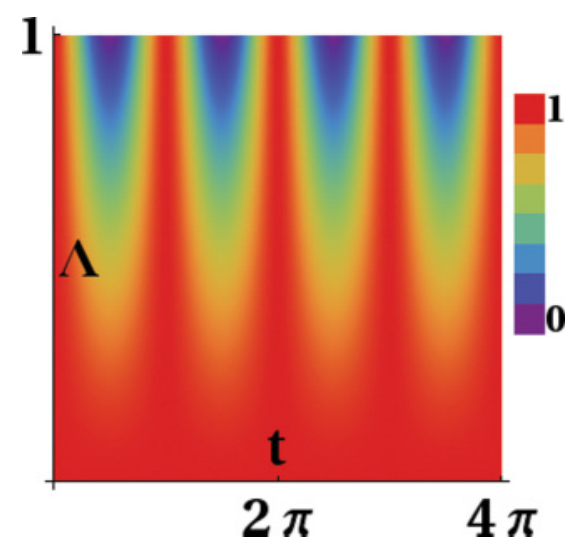

FIG. 5. (Color online) Density plot showing the behavior of the absolute value of the autocorrelation function plotted against normalized time $t=\hbar / \tau$ and strength $\Lambda$ for the exact solution at $\kappa=0$. As expected, only for large values of the interaction strength the evolved state departs considerably from the initial state configuration (see main text).

On the contrary, the maxima and minima of spin polarization correspond in this case to quantum suppressions and are shown as blue (dark) zones in the figure. As soon as we move toward finite values of $\kappa$ (see Fig. 6) interference phenomena come again into play, and we find recurrences represented as read (dark gray) zones and suppressions, described by purple (black) zones. These arise because of the constructive and destructive interference effects, described previously, and are modulated by the ac driving. To check that this physics is different from the static scenario, in Figs. 7 and 8, we depict the corresponding contour plots for time-independent RSOC interaction. For the $\kappa=0$, case shown in Fig. 7, the alternating pattern of quantum revivals and suppressions is separated by the inverse of the energy gap $\delta_{0}$. This is due to the fact that in this situation, the relative phase among the noninteracting eigenstates is set by the energy separation among them, i.e., $\delta_{0}$, and according to Heisenberg's time-energy uncertainty relation, one should have $\delta t$ inversely proportional to the energy separation. As soon as $\kappa$ is finite, a quasihomogeneous

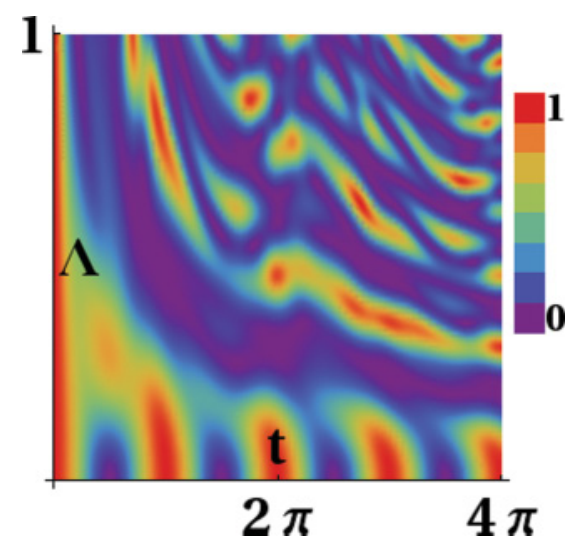

FIG. 6. (Color online) Density plot showing the behavior of the absolute value of the autocorrelation function against normalized time $t=\hbar / \tau$ and RSOC strength $\Lambda$ for the Magnus-Floquet solution at $\kappa=1$. The phase interference effects previously discussed. Now the onset of recurrences at small $\Lambda$ is a consequence of interlevel mixing.

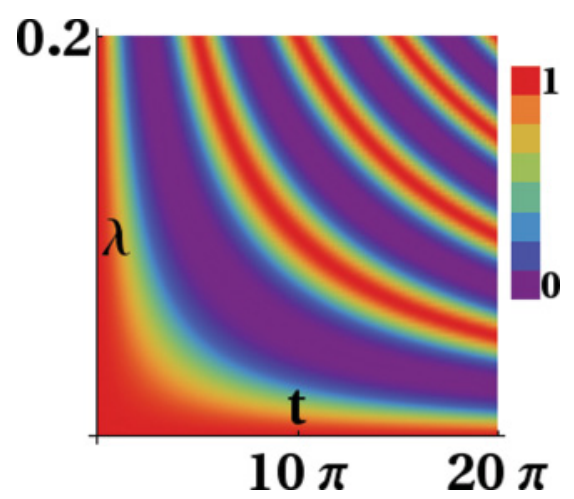

FIG. 7. (Color online) Density plot showing the behavior of the absolute value of the autocorrelation function vs normalized time and RSOC strength $\Lambda$ for the static interaction at $\kappa=0$. In this case, quantum revivals are separated by the inverse of the gap $\delta_{0}=2 \lambda$ in accordance to Heisenberg uncertainty principle.

pattern of recurrences is seen. Therefore although in the static case where the spin polarization vanishes for all values of RSOC strength, there appear recurrences indicating there is no longer correlation between $S_{z}$ and $A(t)$, which for the system under study are only correlated for time-dependent driving.

Two comments are in order here. The first one is concerning the validity of the Dirac fermion Hamiltonian approximation in order to deal with spin-orbit related phenomena in graphene monolayer. As it is discussed in Ref. 21, the large gap of the Hamiltonian describing the $\sigma$ orbitals implies that the effective Hamiltonian including SOC is essentially given by the longwavelength or Dirac Hamiltonian considered in our model. In addition, M. Gmitra et al. ${ }^{23}$ used first-principles calculations to discuss the relevance of spin-orbit related physics in graphene near and beyond the Dirac points. The second point to remark is the role of localized impurities. As has been discussed in Ref. 24, the presence of local impurities can enhance the value of the static intrinsic spin-orbit coupling strength because of the induced $s p^{3}$ distortion that leads to a hybridization of the $\pi$ and $\sigma$ orbitals and as shown in Ref. 23, RSOC only respond to

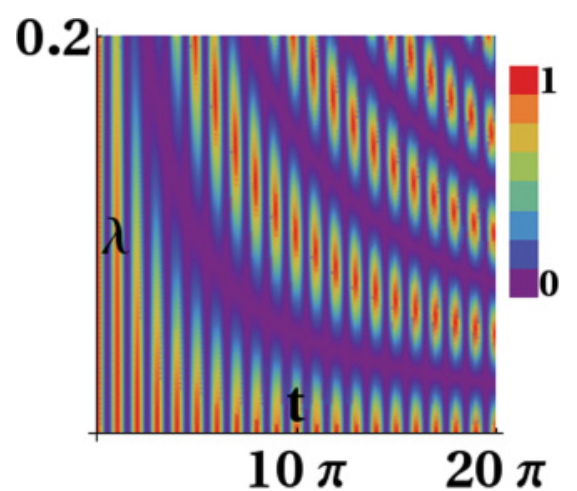

FIG. 8. (Color online) For finite values of momentum $\kappa=1$, recurrences are modulated signaling the coexistence of revivals for times inversely proportional to the static gap $\delta_{0}=2 \lambda$ and those corresponding to the larger energy separation $\delta=\sqrt{k^{2}+\lambda^{2}}$, which correspond to a smaller time and seen in the figure as modulated alternating pattern of maxima and minima for each recurrence curve corresponding to $k=0$. 
this $\sigma-\pi$ hybridization. Therefore we would expect our results to be robust or even enhanced if localized impurities were included in the model.

Now we would like to compare to other proposals of dynamical modulation of energy gaps under ac driven in graphene. For instance, Oka and Aoki ${ }^{25}$ found that circularly polarized intense laser fields can induce a photovoltaic effect in graphene, that is, a Hall effect without magnetic fields. This, in turn, relies on the gap opening at the Dirac point $k=0$. However, as shown recently by $\mathrm{Zhou}$ and $\mathrm{Wu}^{26}$ in analyzing the optical response of graphene under intense THz fields, the contributions from large momenta make the effective gaps opened to get also dynamically closed. However, it is found that the quasienergy spectrum for the linear polarization leads to linear quasienergy spectrum (see also Ref. 27). Our model could, in principle, be mapped into their scenario of linearly polarized radiation field. Yet, in both works, ${ }^{26,27}$ the case of linear polarization leads to linear quasienergy spectrum. Therefore, from the semiparabolic quasienergy spectrum shown in Fig. 1, we can infer that the bending of the quasienergy spectrum makes the spin-orbit driven scenario qualitatively different from these other approaches to dynamical control of graphene electronic properties. Our argument relies on the fact that the topological properties of periodically driven systems are characterized by the quasienergy spectrum, ${ }^{15}$ so we can conclude that the physics related to ac-driven spin orbit does reveal new physical interesting electronics properties that are absent in the static regime.

\section{CONCLUSIONS}

We have investigated the role of periodically driven RSOC in monolayer graphene and recasted the original fourdimensional problem as an equivalent set of two two-level problems. Due to the induced modulation of the relative phases among the static eigenstates, we found a closing of the static gap at the Dirac point $k=0$. This result is in agreement with the available exact solution and differs from RWA where an unphysical gap is seen to appear. This physical picture is confirmed through a Fourier mode expansion and we found that Magnus Floquet approach indeed has the advantage of providing the quasienergy spectrum with less computational effort. We also found that the generation and manipulation of out-of-plane spin polarization for otherwise spin-unpolarized states requires the time driving to be realizable within this set up. Due to the induced interlevel mixing among the static eigenstates, we found a set of alternating positive and negative spin phases in clear distinction to the well separated spin phases at the Dirac point corresponding to the exact solution. The dynamical onset of quantum revivals described through the autocorrelation function is directly correlated to the appearance of maxima for either spin phases. However, in the static case, such a correlation does not ensue since the spin polarization vanishes identically, whereas, the quantum revivals are still present. Concerning the actual experimental realization of our proposal, we believe it could be implemented by means of magnetic resonance force microscopy as reported in Ref. 28. Within this scheme, single-spin polarization could be detected by means of the frequency shift induced on a cantilever that is used to scan the sample. The sign of the cantilever's frequency shift can be associated to the spin polarization. This detection is achieved by means of low-intensity magnetic fields under resonant conditions, thus no magnetic coupling terms need to be included in the description of the dynamics of the charge carriers in graphene. In this way, although, in principle, RSOC in graphene has a small static strength, the time-dependent effective phenomena produce some interesting spin controlling strategies, which we believe could provide a route to new implementations of graphene in spintronic devices with appropiate spin detection techniques.

\section{ACKNOWLEDGMENTS}

Z.Z.S. thanks the Alexander von Humboldt Foundation (Germany) for a grant and A.L. thanks DAADFUNDAYACUCHO for financial support. This work has been supported by Deutsche Forschungsgemeinschaft via GRK 1570 .

\section{APPENDIX: DERIVATION OF ITERATIVE TERMS AND QUASIENERGY SPECTRUM}

Using the simplifying notation for the time-dependent interaction $h^{-}\left(k, t_{i}\right)=h_{i}^{-}$, one gets to fourth order in the iteration procedure that

$$
\begin{gathered}
F_{1}=\frac{-i}{T \hbar} \int_{0}^{T} h_{1}^{-} d t_{1}, \\
F_{2}=\frac{-i^{2}}{2 T \hbar^{2}} \int_{0}^{T} d t_{1} \int_{0}^{t_{1}}\left[h_{1}^{-}, h_{2}^{-}\right] d t_{2}, \\
F_{3}=\frac{-i^{3}}{6 T \hbar^{3}} \int_{0}^{T} d t_{1} \int_{0}^{t_{1}} d t_{2} \int_{0}^{t_{2}}\left(\left[h_{1}^{-},\left[h_{2}^{-}, h_{3}^{-}\right]\right]\right. \\
\left.+\left[h_{3}^{-},\left[h_{2}^{-}, h_{1}^{-}\right]\right]\right) d t_{3}, \\
F_{4}=\frac{-i^{4}}{12 T \hbar^{4}} \int_{0}^{T} d t_{1} \int_{0}^{t_{1}} d t_{2} \int_{0}^{t_{2}} d t_{3} \int_{0}^{t_{3}}\left(\left[\left[\left[h_{1}^{-}, h_{2}^{-}\right], h_{3}^{-}\right], h_{4}^{-}\right]\right. \\
+\left[\left[\left[h_{3}^{-}, h_{2}^{-}\right], h_{4}^{-}\right], h_{1}^{-}\right]+\left[\left[\left[h_{3}^{-}, h_{4}^{-}\right], h_{2}^{-}\right], h_{1}^{-}\right] \\
\left.+\left[\left[\left[h_{4}^{-}, h_{1}^{-}\right], h_{3}^{-}\right], h_{2}^{-}\right]\right) d t_{4},
\end{gathered}
$$

and performing the corresponding calculations one gets

$$
\begin{gathered}
F_{1}=\frac{i \kappa \Lambda}{\sqrt{\kappa^{2}+\Lambda^{2}}}\left(\kappa \sigma_{z}+\Lambda \sigma_{x}\right), \\
F_{3}=\frac{-i \kappa \Lambda}{\sqrt{\kappa^{2}+\Lambda^{2}}}\left[5 \kappa \Lambda \sigma_{z}-\left(4 \kappa^{4}-\Lambda^{2}\right) \sigma_{x}\right], \\
F_{5}=\frac{i \kappa \Lambda}{36 \sqrt{\kappa^{2}+\Lambda^{2}}}\left[7 \kappa \Lambda\left(-144 \kappa^{2}+31 \Lambda^{2}\right) \sigma_{z}\right. \\
\left.+\left(576 \kappa^{4}-640 \kappa^{2} \Lambda^{2}+9 \Lambda^{4}\right) \sigma_{x}\right],
\end{gathered}
$$


whereas the even contributions $F_{2 j}$ all vanish. The quasienergies $\varepsilon_{ \pm}$are obtained through diagonalization of the self-adjoint matrix $-i F=-i \sum_{j} F_{j}$, and up to this order, one gets

$$
\varepsilon_{ \pm}= \pm \frac{1}{36}\left[\kappa^{2}\left(331776 \kappa^{6} \Lambda^{2}+81\left(\Lambda^{2}-2\right)^{4} \kappa^{4}\left(23 \Lambda^{4}-72 \Lambda^{2}\right)+32 \kappa^{2}\left(1109 \Lambda^{6}-900 \Lambda^{4}-324 \Lambda^{2}\right)\right]^{1 / 2} .\right.
$$

*To whom correspondence should be addressed: alexander.lopez@physik.uni-regensburg.de

${ }^{1}$ K. S. Novoselov, A. K. Geim, S. V. Morozov, D. Jiang, Y. Zhang, S. V. Dubonos, I. V. Grigorieva, and A. A. Firsov, Science 306, 666 (2004).

${ }^{2}$ A. K. Geim and K. S. Novoselov, Nat. Mater. 6, 183 (2007).

${ }^{3}$ A. H. Castro Neto, F. Guinea, N. M. R. Peres, K. S. Novoselov, and A. K. Geim, Rev. Mod. Phys. 81, 109 (2009).

${ }^{4}$ S. Das Sarma, S. Adam, E. H. Hwang, and E. Rossi, Rev. Mod. Phys. 83, 407 (2011).

${ }^{5}$ C. L. Kane and E. J. Mele, Phys. Rev. Lett. 95, 226801 (2005).

${ }^{6}$ H. Min, J. E. Hill, N. A. Sinitsyn, B. R. Sahu, L. Kleinman, and A. H. MacDonald, Phys. Rev. B 74, 165310 (2006).

${ }^{7}$ D. Huertas-Hernando, F. Guinea, and A. Brataas, Phys. Rev. B 74, 155246 (2006).

${ }^{8}$ T. Stauber and J. Schliemann, New J. Phys. 11, 115003 (2009).

${ }^{9}$ E. I. Rashba, Phys. Rev. B 79, 161409(R) (2009).

${ }^{10}$ C. Ertler, S. Konschuh, M. Gmitra, and J. Fabian, Phys. Rev. B 80, 041405(R) (2009).

${ }^{11}$ D. Huertas-Hernando, F. Guinea, and A. Brataas, Phys. Rev. Lett. 103, 146801 (2009).

${ }^{12}$ P. Foldi, M. G. Benedict, O. Kalman, and F. M. Peeters, Phys. Rev. B 80, 165303 (2009).

${ }^{13}$ M. Grifoni, P. Hänggi, Phys. Rep. 304, 229 (1998).
${ }^{14}$ S.-I Chu, D. A. Telnov, Phys. Rep. 390, 1 (2004).

${ }^{15}$ T. Kitagawa, E. Berg, M. Rudner, and E. Demler, Phys. Rev. B 82, 235114 (2010).

${ }^{16}$ N. H. Lindner, G. Refael, and V. Galinski, Nat. Phys. 7, 490 (2011).

${ }^{17}$ W. Magnus, Comm. Pure Appl. Math. 7, 649 (1954).

${ }^{18}$ S. Blanes, F. Casas, J. A. Oteo, and J. Ros, Phys. Rep. 470, 151 (2009).

${ }^{19}$ V. Krueckl and T. Kramer, New J. Phys. 11, 093010 (2009).

${ }^{20}$ M. Zarea and N. Sandler, Phys. Rev. Lett. 99, 256804 (2007).

${ }^{21}$ Y. Yao, F. Ye, X. L. Qi, S. C. Zhang, and Z. Fang, Phys. Rev. B 75, 041401 (2007)

${ }^{22}$ Y. S. Dedkov, M. Fonin, U. Rudiger, and C. Laubschat, Phys. Rev. Lett. 100, 107602 (2008).

${ }^{23}$ M. Gmitra, S. Konschuh, C. Ertler, C. Ambrosch-Draxl, and J. Fabian, Phys. Rev. B 80, 235431 (2009).

${ }^{24}$ A. H. Castro Neto and F. Guinea, Phys. Rev. Lett. 103, 026804 (2009).

${ }^{25}$ T. Oka and H. Aoki, Phys. Rev. B 79, 081406(R) (2009).

${ }^{26}$ Y. Zhou and M. W. Wu, Phys. Rev. B 83, 245436 (2011).

${ }^{27}$ H. L. Calvo, H. M. Pastawski, S. Roche, and L. E. F. Foa Torres, Appl. Phys. Lett. 98, 232103 (2011).

${ }^{28}$ D. Rugal, R. Budakian, H. J. Mamin, and B. W. Chui, Nature (London) 430, 329 (2004). 